\title{
Band structure of CuMnAs probed by optical and photoemission spectroscopy
}

\author{
M. Veis, ${ }^{1}$ J. Minár, ${ }^{2}$ G. Steciuk,${ }^{3}$ L. Palatinus,${ }^{3}$ C. Rinaldi, ${ }^{4}$ M. Cantoni, ${ }^{4}$ D. Kriegner, ${ }^{3,1}$ K. K. Tikuišis, ${ }^{1}$ J. Hamrle, ${ }^{1}$ \\ M. Zahradník, ${ }^{1}$ R. Antoš,${ }^{1}$ J. Železný, ${ }^{3}$ L. Šmejkal, ${ }^{3,5}$ X. Marti, ${ }^{3}$ P. Wadley, ${ }^{6}$ R. P. Campion, ${ }^{6}$ C. Frontera, ${ }^{7}$ K. Uhlírová, ${ }^{1}$ \\ T. Duchoň, ${ }^{8}$ P. Kužel, ${ }^{9}$ V. Novák, ${ }^{3}$ T. Jungwirth, ${ }^{3,6}$ and K. Výborný ${ }^{3}$ \\ ${ }^{1}$ Charles University, Faculty of Mathematics and Physics, Ke Karlovu 5, Praha 2, Czech Republic \\ ${ }^{2}$ New Technologies-Research Center University of West Bohemia, Plzeň, Czech Republic \\ ${ }^{3}$ Institute of Physics, Academy of Science of the Czech Republic, Cukrovarnická 10, Praha 6, Czech Republic \\ ${ }^{4}$ Department of Physics, Politecnico di Milano, via G. Colombo 81, 20133 Milano, Italy \\ ${ }^{5}$ Institut für Physik, Johannes Gutenberg Universität Mainz, D-55099 Mainz, Germany \\ ${ }^{6}$ School of Physics and Astronomy, University of Nottingham, Nottingham NG7 2RD, United Kingdom \\ ${ }^{7}$ Institut de Ciència de Materials de Barcelona (ICMAB-CSIC), Campus Universitari de Bellaterra, Cerdanyola del Vallès, 08193 Spain \\ ${ }^{8}$ Charles University, Faculty of Mathematics and Physics, Department of Surface and Plasma Science, V Holešovičkách 2, \\ 18000 Praha 8, Czech Republic \\ ${ }^{9}$ Institute of Physics, Academy of Science of the Czech Republic, Na Slovance 1999/2, Praha 8, Czech Republic
}

(Received 30 November 2017; revised manuscript received 12 February 2018; published 8 March 2018)

\begin{abstract}
The tetragonal phase of CuMnAs progressively appears as one of the key materials for antiferromagnetic spintronics due to efficient current-induced spin-torques whose existence can be directly inferred from crystal symmetry. Theoretical understanding of spintronic phenomena in this material, however, relies on the detailed knowledge of electronic structure (band structure and corresponding wave functions) which has so far been tested only to a limited extent. We show that AC permittivity (obtained from ellipsometry) and UV photoelectron spectra agree with density functional calculations. Together with the x-ray diffraction and precession electron diffraction tomography, our analysis confirms recent theoretical claim [Phys. Rev. B 96, 094406 (2017)] that copper atoms occupy lattice positions in the basal plane of the tetragonal unit cell.
\end{abstract}

DOI: 10.1103/PhysRevB.97.125109

Magnetic moments in antiferromagnets have been notoriously difficult to manipulate. With the exception of materials having low Néel temperature and small magnetic anisotropy, very strong magnetic fields must be applied. Such fields would be too strong to be of any practical use and, moreover, they can never be applied as locally as electric pulses. Recently, an alternative manipulation mechanism has been proposed [1] which relies on current-induced spin-orbit torques (SOTs) acting in the bulk of the antiferromagnetic material. They result from a buildup of staggered spin polarization (i.e., the one which alternates sign on two magnetic sublattices) in response to an applied uniform electric current; such polarization can be calculated in the framework of linear response to electric field [2,3]. A prediction of sizable SOT in CuMnAs has soon been experimentally confirmed [4] and prototype memories where the writing is done using SOT have been demonstrated [5]. Devices based on thin films of CuMnAs thus claim a prominent role within the fast developing field of antiferromagnetic spintronics $[6,7]$.

Quantitative modeling of SOT (and many other materialspecific quantities) relies on a detailed knowledge of the electronic structure [8]. While well-established $a b$ initio methods have been used for this purpose, little effort has so far been dedicated to validating the band structure in terms of comparing calculated and measured spectral properties [9]. We fill this gap by exploring the complex AC permittivity in the optical range and photoemission spectroscopy in the UV range (UPS) and comparing them to density functional theory (DFT) calculations. We find a good agreement between the experimental data and the calculated properties provided the electronic correlations are treated beyond DFT, using Hubbard correction characterized by an on-site repulsion $U$ on $\mathrm{Mn} 3 d$ orbitals. Moreover, we demonstrate that the AC permittivity in the optical range can be used to discern different phases of CuMnAs. Focusing on the tetragonal phase of CuMnAs [10], we corroborate analysis of our spectral measurements by precession electron diffraction tomography (PEDT), which points to a phase recently claimed to have the lowest theoretically calculated total energy [11].

The studied thin films of CuMnAs were prepared by molecular beam epitaxy (MBE; we followed procedures described in Ref. [10]). We performed the standard x-ray diffraction (XRD) structural characterization and PEDT. Ellipsometry was carried out on a nominally 20-nm-thick layer, while PEDT was applied to 150-nm-thick layers, both grown on a $\mathrm{GaP}(001)$ substrate. Photoemission spectra in the UV range (UPS) were obtained for a 130-nm-thick sample grown on a $\mathrm{GaAs}(001)$ substrate.

Both XRD and PEDT confirm the tetragonal crystal structure shown in Fig. 1 with space group $P 4 / \mathrm{nmm}$. Regarding the occupancy of lattice sites labeled $S^{1}, S^{2}$, and $S^{3}$ in the figure, neither $\mathrm{x}$-ray nor electron diffraction are very efficient in distinguishing manganese and copper atoms because of their similar scattering powers; the latter method, however, does provide some advantage over the former one as we show below. In this paper, we consider theoretically two tetragonal phases which are defined as follows: The first structure has copper atoms at the basal positions $S^{1}$ (Wyckoff position 2a) 

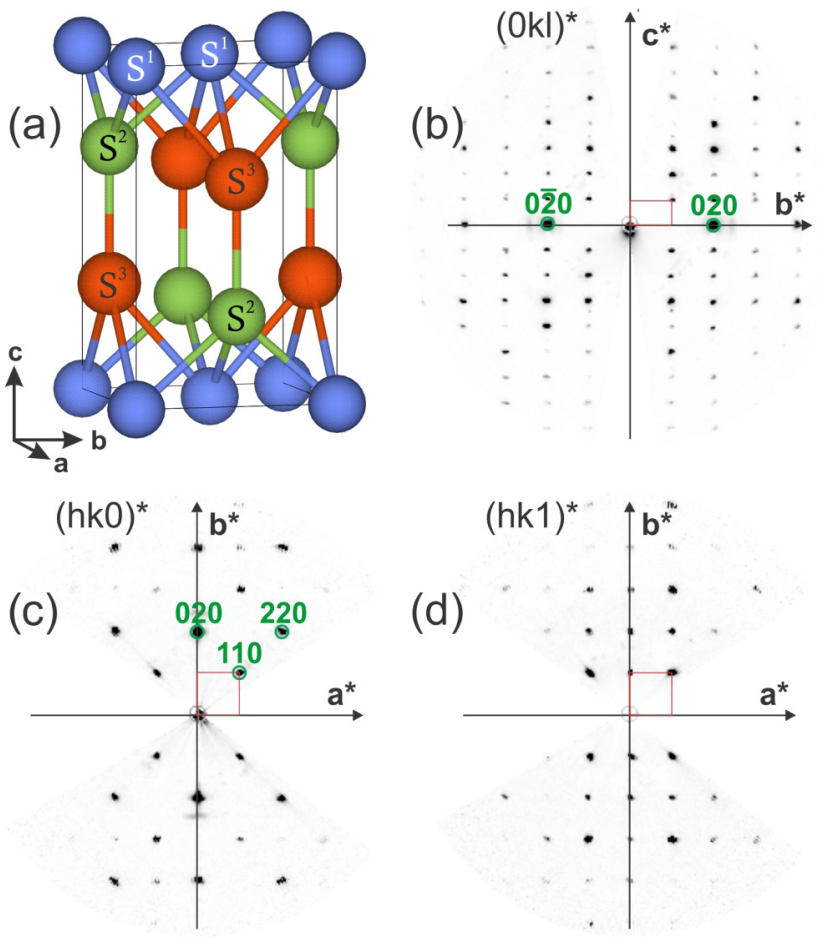

(d)

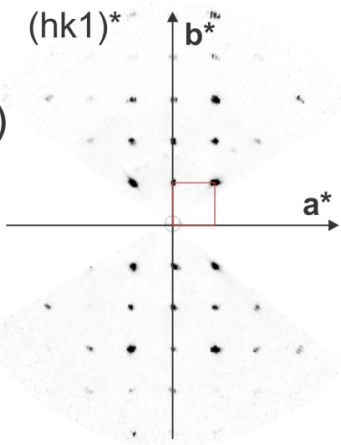

FIG. 1. (a) Structure of the tetragonal phase of CuMnAs. In RTP, $\mathrm{Cu} / \mathrm{As} / \mathrm{Mn}$ atoms occupy the sites $S^{1} / S^{2} / S^{3}$. In the inverted phase, $\mathrm{Cu}$ and $\mathrm{Mn}$ atoms are swapped. (b)-(d) Electron diffraction (PEDT) patterns: $(0 \mathrm{kl})^{*},(\mathrm{hk} 0)^{*}$, and $(\mathrm{hk} 1)^{*}$ sections of the reciprocal space reconstructed from PEDT data (PETS program [13]). The conditions $h+k=2 n$ on $h k 0, h=2 n$ on $h 00$ and $k=2 n$ on $0 k 0$ are characteristic of the $n$ glide.

and As/Mn at $S^{2} / S^{3}$ (Wyckoff position $2 c$ ). We will refer to this as the reference tetragonal phase (RTP). The second, inverted structure is obtained by swapping $\mathrm{Mn}$ and $\mathrm{Cu}$ so that the basal positions $S^{1}$ are occupied by manganese. Regarding the magnetic structure of the latter phase, we only consider the case of antiferromagnetic ordering within the basal plane where the unit cell contains six atoms.

Using XRD, we find lattice constants differing by less than $1 \%$ for samples grown on $\mathrm{GaP}$ and GaAs (e.g., $a=0.3853 \mathrm{~nm}$ and $a=0.3820 \mathrm{~nm}$ at room temperature, respectively). For effects considered in this work, such differences lead to negligible changes in observed (optical and photoelectron emission) spectra, which renders, within the scope of this paper, all our thin film samples interchangeable. Further details about x-ray characterization can be found in the Supplemental Material [12] (Sec. I) and we now turn our attention to the electron diffraction analysis.

For PEDT characterization, a cross section of the $\mathrm{CuMnAs} / \mathrm{GaP}(001)$ thin film was prepared by mechanic polishing followed by ion milling. Four PEDT data sets were recorded on several parts of the film using a Philips CM120 electron transmission microscope $\left(V_{a c c}=120 \mathrm{kV}, \mathrm{LaB}_{6}\right)$ with the precession device Nanomegas Digistar and a side-mounted CCD camera Olympus Veleta with 14bit dynamic range. The precession angle and the tilt step of the goniometer were both set to 1 degree. The data were analyzed using the computer programs PETS [13] and JANA2006 [14].
The tetragonal structure as shown in Fig. 1(a) was confirmed by the PEDT data. Extinction conditions observed on the sections of the reciprocal space shown in Figs. 1(b)-1(d) are compatible with the space group $P 4 / \mathrm{nmm}$. RTP was used as a starting structure and refined from the PEDT data using the dynamical theory of diffraction ("dynamical refinement") according to Refs. [15,16]. All four PEDT data sets were combined to increase the statistics of the refinement and the coverage of the reciprocal space. Results of the refinement are summarized in Table I. We measured over four thousand reflections in all data sets $\left(\mathrm{N}_{\text {all }}\right)$ and found $\mathrm{N}_{\mathrm{obs}}$ reflections with a significant intensity. Among model parameters, there are seven structural parameters (two $z / c$ parameters, three displacement parameters, and two occupancy factors), an average thickness of the analyzed area for each of the four datasets and one scaling parameter per each experimental diffraction pattern giving in total $\mathrm{N}_{\text {param }} \ll \mathrm{N}_{\text {obs }}$ optimized parameters. Note that datato-parameter ratio $\mathrm{N}_{\text {all }} / \mathrm{N}_{\text {param }}>10$ is required for a reliable structure determination. The quality of the fit is demonstrated by the $R$ value [17] of 10.46 and only slightly larger weighted $R$ value. For atomic positions $z / c$, we obtain values in a good agreement with the corresponding values inferred from $\mathrm{X}$-ray analysis [12]. The occupancy of $S^{3}$ is found significantly different from one suggesting that our samples are copper rich.

The key added value of PEDT in the context of this study is the ability to better distinguish RTP from the inverted structure, and to this end, the isotropic displacement parameters $\mathrm{U}_{\text {iso }}\left(S^{1}\right)$ and $\mathrm{U}_{\text {iso }}\left(S^{3}\right)$ (also known as ADP) are the most sensitive indicators. If atomic types are correctly assigned to individual atomic positions, their values should be approximately equal. For the $S^{1}$ and $S^{3}$ sites in Fig. 1, the ADPs in the RTP model do have similar values, consistent with previous studies $[18,19]$. However, they change unfavorably for the inverted tetragonal phase: The ADP drops (increases) by about $65 \%$ for the $S^{1}$ $\left(S^{3}\right)$ site, respectively. This result is consistent with the higher electron atomic scattering amplitude of $\mathrm{Cu}\left(f_{\mathrm{Cu}}^{B}\right)$ relative to $\mathrm{Mn}\left(f_{\mathrm{Mn}}^{B}\right)$. In other words, RTP seems more consistent with the electron diffraction data. Note that also the $R$ value in Table I for the inverted structure is appreciably larger than for RTP. From this point on, we will focus on the RTP and will not consider the inverted structure (phase) unless explicitly stated.

A Mueller matrix ellipsometer JA Woollam RC2 was employed to acquire experimental spectra of ellipsometric parameters $\Psi$ and $\Delta$. To ensure a sufficiently large ensemble of experimental data necessary for fitting, spectra were measured at several angles of incidence $\left(55^{\circ}, 60^{\circ}, 65^{\circ}, 70^{\circ}\right)$. The experimental data were fitted using the Woollam CompleteEase software starting with a model structure of nominally 20-nm-thick CuMnAs layer on GaP substrate and a surface oxide layer was accounted for, which naturally occurs when the sample is exposed to air (see Sec. II of Supplemental Material [12] for details). Optical constants of GaP were taken from literature [20], while the permittivity of CuMnAs was parametrized by a combination of Drude, Tauc-Lorentz, and three Lorentz functions. All parameters were fitted together with the layer thickness $\left(l_{\mathrm{CuMnAs}}\right)$ and surface roughness. The resulting $l_{\mathrm{CuMnAs}}=22.6 \mathrm{~nm}$ along with a negligible surface roughness confirm the high level of sample growth control. Also, the mean square error (MSE) was lower than 1, 
TABLE I. Crystallographic and dynamical refinement parameters of the RTP and the inverted tetragonal phases, both of $P 4 / \mathrm{nmm}(\mathrm{No} .129)$ space group. For RTP: $\mathrm{Cu}\left(S^{1}\right)$ occupies the Wyckoff position $2 \mathrm{a}\left(\frac{1}{2}, \frac{1}{2}, 0\right), \mathrm{Mn}\left(S^{3}\right)$ and As $\left(S^{2}\right)$ occupy positions $2 \mathrm{c}\left(0, \frac{1}{2}, z\right)$. In the inverted tetragonal phase $S^{1}=\mathrm{Mn}$ and $S^{3}=\mathrm{Cu}$.

\begin{tabular}{|c|c|c|c|c|c|c|c|c|}
\hline \multicolumn{9}{|c|}{ Structural parameters: } \\
\hline & \multicolumn{2}{|c|}{$z / c$} & \multicolumn{3}{|c|}{ occupancies } & \multicolumn{3}{|c|}{ ADPs (iso.) $\left[\AA^{2}\right]$} \\
\hline & $S^{2}$ & $S^{3}$ & $S^{1}$ & $S^{2}$ & $S^{3}$ & $S^{1}$ & $S^{2}$ & $S^{3}$ \\
\hline RTP: & $0.2627(2)$ & $0.6628(2)$ & $0.995(8) \sim 1$ & 1 & $0.869(7)$ & $0.0147(4)$ & $0.0121(3)$ & $0.0123(4)$ \\
\hline inverted: & $0.2627(2)$ & $0.6624(2)$ & $0.870(6)$ & 1 & $0.949(8)$ & $0.0052(4)$ & $0.0124(3)$ & $0.0205(5)$ \\
\hline \multicolumn{9}{|c|}{ Refinement parameters: } \\
\hline RTP: & \multirow{2}{*}{\multicolumn{2}{|c|}{$\begin{array}{l}\mathrm{N}_{\text {param. }}=338 ; \\
N_{\text {nam }}=338 .\end{array}$}} & \multicolumn{2}{|c|}{$\mathrm{N}_{\mathrm{obs} / \mathrm{all}}=3768 / 4189$} & \multicolumn{2}{|c|}{$\mathrm{R}_{\mathrm{obs}}=10.15$} & \multicolumn{2}{|c|}{$\mathrm{wR}_{\mathrm{all}}=11.86$} \\
\hline inverted: & & & \multicolumn{2}{|c|}{$\mathrm{N}_{\mathrm{obs} / \mathrm{all}}=3767 / 4190$} & \multicolumn{2}{|c|}{$\mathrm{R}_{\mathrm{obs}}=10.68$} & \multicolumn{2}{|c|}{$\mathrm{wR}_{\mathrm{all}}=12.51$} \\
\hline
\end{tabular}

implying a rather robust fit whose result is shown in Fig. 2 as experimental data.

Our DFT $+U$ calculations [23] for tetragonal CuMnAs ( $a=0.3853 \mathrm{~nm}, c=0.6276 \mathrm{~nm}$ ) based on generalized gradient approximation (GGA) with scalar-relativistic correction come quite close to the experimental data (Fig. 2), provided relatively large broadening $(\Gamma=0.7 \mathrm{eV})$ of the interband terms is used ( $n \neq n^{\prime}$ in Eq. (16) of Ref. [24]). Such value is not unprecedented [25] although still significantly larger than $\hbar / 2 \tau$ implied by Drude-formula relaxation time $\tau$ obtained from measured DC conductivity. That said, one should be reminded that the intra- and interband relaxation times are not required to be the same so that parameters used for the model in Fig. 2 are still plausible. To estimate $\tau$, we used (apart from the experimental resistivity [10]) the ab initio calculated plasma frequency $\omega_{p}$. The model data plotted in Fig. 2 also include the intraband contribution (Drude peak). For the sake of definiteness, we should mention that they correspond to $\epsilon_{x x}$ where $x$ and Mn magnetic moments lay in the $a b$ plane as defined in Fig. 1(a). In the remainder of this paper, we will only be discussing the imaginary part of permittivity since the Kramers-Kronig-related real part bears no additional information.

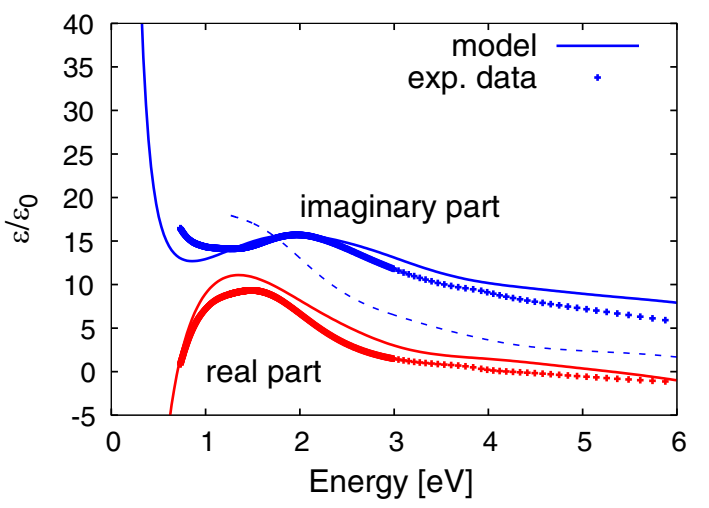

FIG. 2. AC permittivity of CuMnAs thin film determined by ellipsometry (for comparison, $\operatorname{Im} \epsilon / \epsilon_{0}$ for orthorhombic bulk CuMnAs grown by other method [21] is shown by the dashed line; see Supplemental Material [12], Sec. III for growth details [22]). The GGA $+U$ model uses $U=1.7 \mathrm{eV}$ and $\Gamma=0.7 \mathrm{eV}$ for the interband part and $\hbar \omega_{p}=3.26 \mathrm{eV}$ and $\hbar / 2 \tau=120 \mathrm{meV}$ for the intraband contribution.
Accounting for electron correlations turns out to be essential. We use GGA $+U$ with double-counting corrections to the DFT part treated in the fully localized limit [26] (FLL) and find the peak in the imaginary part of the permittivity blueshifting with increasing value of $U$ (see Fig. 3). Its experimentally determined position $(\hbar \omega \approx 2 \mathrm{eV})$ is recovered for $U=1.7 \mathrm{eV}$, and on the theoretical side, the peak stems from unoccupied Mn states (indicated by an arrow in the inset of Fig. 3). In Fig. 4, the corresponding band structure is shown. At this point, we remark that the inverted phase has a markedly different band structure, but its optical response still resembles the experimental data in Fig. 2 provided unrealistically large $U$ is chosen. Returning to the RTP, we now also briefly discuss the effect of the parameter [26] $J>0$. It causes the peak in $\operatorname{Im} \epsilon / \epsilon_{0}$ to shift to lower energies (in agreement with replacing $U$ and $J$ by $U_{\text {eff }}=U-J$ and $J=0$ ), and also it adds some additional structure to the peak. The large broadening, however, renders such effects unobservable. Based on ellipsometry data, values of $U-J \approx 2 \mathrm{eV}$, therefore, seem to give the best results.

Photoemission spectra and also inverse photoemission spectra (IPES) were measured for CuMnAs thin layers covered originally (after growth) by an arsenic cap. This protective layer was removed by Ar ion milling in the UHV environment for UPS and IPES [27]. The cleanness of the surface was

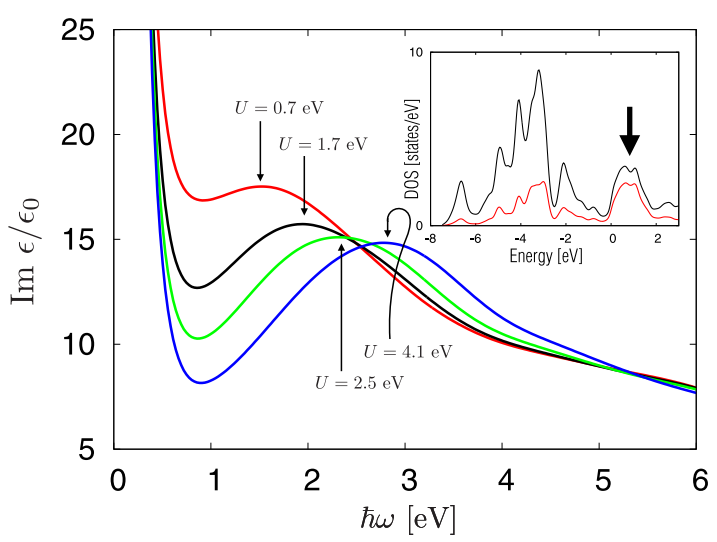

FIG. 3. The imaginary part of relative permittivity calculated for several values of $U$. The peak shifts blue with increasing $U$, the arrows indicate the position of the maximum. Inset: density of states per spin (and Mn-partial density of states shown in red) with a Mn-dominated peak just above the Fermi level (taken as $E=0$ ). 


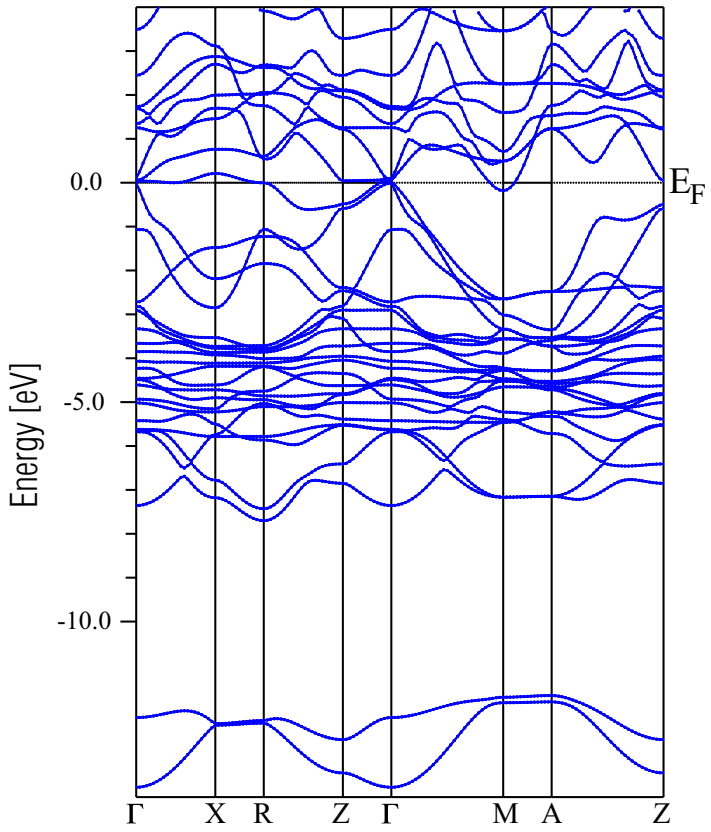

FIG. 4. Based on the comparison between ellipsometry and $\mathrm{GGA}+U$ calculations [23], this band structure $(U=1.7 \mathrm{eV})$ seems to describe well CuMnAs in tetragonal phase.

checked in situ by x-ray photoemission spectroscopy (XPS): The disappearance of core-level peaks $\mathrm{O} 1 \mathrm{~s}$ and $\mathrm{C} 1 \mathrm{~s}$ indicates that the surface is clean (the residual contamination is well below $1 \%$ of surface coverage). The UPS spectrum, shown in Fig. 5 by black squares, was recorded using a helium lamp as excitation source $(\mathrm{HeI}-\alpha=21.2 \mathrm{eV})$ and a hemispherical energy analyzer Phoibos 150 (SPECS ${ }^{\mathrm{TM}}$ ), with an acceptance angle of $\sim 6^{\circ}$ and a field view of $1.4 \mathrm{~mm}^{2}$. Results of the investigation of empty states above the Fermi level by IPES is shown only in the Supplemental Material [12] (Section IV). The calculated DOS above the Fermi level is less sensitive to variations of $U$ and, moreover, fine details cannot be accessed by IPES because of the large experimental broadening [28] characteristic of these spectra.

Photoemission spectroscopies access the electronic structure associated with top $\approx 1 \mathrm{~nm}$ of the thin layer [29]. In

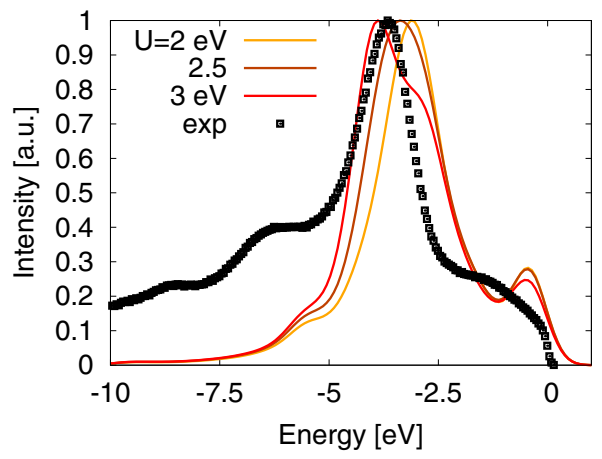

FIG. 5. Experimental angle-integrated photoemission (UPS, black squares) compared to the corresponding one-step model of photoemission. Theoretical data are shown for three values of $U$; labeled features are described in the text. the simplest approximation, the measured angle-integrated UPS and IPES should reflect rather directly the DOS. This approximation works reasonably well in the high energy regime (XPS) and led [30] to a larger estimate of $U$ around $4.5 \mathrm{eV}$. However, this approach ignores the influence of specific matrix elements that, in general, introduce an energy- and element-dependent weight to DOS. Also, in the regime of low photon energies (as measured here), additional aspects may have a very pronounced impact on the angle-integrated photoemission spectra (for example, final states or surface effects).

Here we used the recently developed full spin-density matrix formulation for the photocurrent $[31,32]$ in the onestep model [33,34] (see details in Supplemental Material [12]) within the relativistic Korringa-Kohn-Rostoker Green function method. This method is implemented in the SPR-KKR program package [35]. Regarding the value of $U$, we arrive at a somewhat different conclusion than what was made in Ref. [30]. Nevertheless, the DOS shown in the inset of Fig. 3 still provides a good means for interpreting, on an elementary level, both the calculated and the measured spectra. They are dominated by the $\mathrm{Mn}$ states located at $\approx 1 \mathrm{eV}$ and $-4 \mathrm{eV}$ (with respect to the Fermi level), the latter having a significant admixture of $\mathrm{Cu}$ states. The peak at $\approx-2 \mathrm{eV}$ with dominantly $\mathrm{Cu}$ character is not visible in the UPS spectra, being probably hidden in the main peak of the measured data. The three main features in experimental spectra are labeled by capital letters in Fig. 5. It turns out that the main strong peak (A), both in terms of its position and width, serves as the best test for calculated spectra and their dependence of the value of $U$. As this value increases, the peak blueshifts and broadens and the best match with the experiment occurs around $U=2.5 \mathrm{eV}$. For larger $U$, a shoulder develops in model calculations which is absent in experimental data while for smaller $U$ the good agreement of peak position is lost. Both this feature and (C) which is also clearly visible in the model calculations, can be backtracked to the Mn $d$ states which are shifted to higher binding energies when $U$ increases. The broad peak (B) located close to the Fermi level shows a strong surface character. We confirmed this theoretically by modifying the surface barrier [36] (see Sec. IV. in Supplemental Material [12]). Given that the surface was probably damaged by ion milling used to remove the cap, only little information about the bulk electronic structure can be extracted from this part of UPS. Finally, regarding the inverted structure, we find that the photoemission spectra qualitatively differ from experimental data if we use band structure consistent with ellipsometry. The level of agreement between UPS data and DFT $+U$ calculations for the RTP suggests that it is this structure and the aforementioned $U-J \approx 2 \mathrm{eV}$ which provide a plausible input for the band structure calculations.

In conclusion, we presented optical spectra of the complex permittivity and photoemission spectra in the UV regime (UPS) of MBE-grown thin layers of CuMnAs, which crystallize in the tetragonal structure, and demonstrated a good level of agreement with the DFT $+U$ calculations. Together with the dynamically refined precession electron diffraction tomography, this agreement strongly suggests that copper occupies the basal positions of the structure ( $S^{1}$ in Fig. 1); on the other hand, calculations of UPS and optical permittivity in the inverted structure cannot be reconciled with one particular 
band structure. Conclusions of the recent theoretical study of Máca et al. [11] regarding the site occupation in the tetragonal phase of CuMnAs agree with our findings.

We acknowledge support from National Grid Infrastructure MetaCentrum provided under the programme "Projects of Large Research, Development, and Innovations Infrastructures" (CESNET LM2015042); Grant Agency of the Czech Republic under Grant No. 15-13436S; CEDAMNF
(CZ.02.1.01/0.0/0.0/15_003/0000358) of the Czech ministry of education (MŠMT) as well as its LM2015087 and LNSMLNSpin grants; Cariplo Foundation, Grant No. 2013-0726 (MAGISTER); Spanish MINECO under MAT2015-67593-P project and the 'Severo Ochoa' Programme (SEV-2015-0496); EU FET Open RIA Grant No. 766566; Engineering and Physical Sciences Research Council Grant No. EP/P019749/1. P.W. acknowledges support from the Royal Society through a University Research Fellowship.
[1] J. Železný, H. Gao, K. Výborný, J. Zemen, J. Mašek, A. Manchon, J. Wunderlich, J. Sinova, and T. Jungwirth, Phys. Rev. Lett. 113, 157201 (2014).

[2] V. M. Edelstein, Solid State Commun. 73, 233 (1990).

[3] B. A. Bernevig and O. Vafek, Phys. Rev. B 72, 033203 (2005).

[4] P. Wadley, B. Howells, J. Železný, C. Andrews, V. Hills, R. P. Campion, V. Novák, K. Olejník, F. Maccherozzi, S. S. Dhesi, S. Y. Martin, T. Wagner, J. Wunderlich, F. Freimuth, Y. Mokrousov, J. Kuneš, J. S. Chauhan, M. J. Grzybowski, A. W. Rushforth, K. W. Edmonds, B. L. Gallagher, and T. Jungwirth, Science 351, 587 (2016).

[5] K. Olejník, V. Schuler, X. Marti, V. Novák, Z. Kašpar, P. Wadley, R. P. Campion, K. W. Edmonds, B. L. Gallagher, J. Garces, M. Baumgartner, P. Gambardella, and T. Jungwirth, Nat. Commun. 8, 15434 (2017).

[6] V. Baltz, A. Manchon, M. Tsoi, T. Moriyama, T. Ono, and Y. Tserkovnyak, Rev. Mod. Phys. 90, 015005 (2018).

[7] T. Jungwirth, X. Marti, P. Wadley, and J. Wunderlich, Nat. Nanotech. 11, 231 (2016).

[8] Hang Li, H. Gao, L. P. Zârbo, K. Výborný, Xuhui Wang, I. Garate, F. Doğan, A. Čejchan, J. Sinova, T. Jungwirth, and A. Manchon, Phys. Rev. B 91, 134402 (2015).

[9] A notable exception is the calculation of magnetic linear dichroism in the x-ray range (see Fig. S2 in the supporting material of Ref. [4]). While these measurements allow for the identification of magnetic moment orientation, the spectral form is not in complete agreement with model calculations.

[10] P. Wadley, V. Novák, R. P. Campion, C. Rinaldi, X. Martí, H. Reichlová, J. Železný, J. Gazquez, M. A. Roldan, M. Varela, D. Khalyavin, S. Langridge, D. Kriegner, F. Máca, J. Mašek, R. Bertacco, V. Holý, A. W. Rushforth, K. W. Edmonds, B. L. Gallagher, C. T. Foxon, J. Wunderlich, and T. Jungwirth, Nat. Commun. 4, 2322 (2013).

[11] F. Máca, J. Kudrnovský, V. Drchal, K. Carva, P. Baláž, and I. Turek, Phys. Rev. B 96, 094406 (2017).

[12] See Supplemental Material at http://link.aps.org/supplemental/ 10.1103/PhysRevB.97.125109 for details on X-ray characterization (Sec. I), copper oxide layer forming at the sample surface (Sec. II), bulk orthorhombic sample (Sec. III) and UPS/IPES measurements (Sec. IV).

[13] L. Palatinus, PETS - program for analysis of electron diffraction data, Institute of Physics of the AS CR, Prague, Czech Rep. (2011).

[14] V. Petříček, M. Dušek, and L. Palatinus, Z. Kristallogr. 229, 345 (2014).

[15] L. Palatinus, V. Petříček, and C. A. Corrêa, Acta Crystallogr. Sect. A 71, 235 (2015).
[16] L. Palatinus, C. A. Corrêa, G. Steciuk, D. Jacob, P. Roussel, P. Boullay, M. Klementová, M. Gemmi, J. Kopeček, M. C. Domeneghetti, F. Cámara, and V. Petříček, Acta Crystallogr. Sect. B 71, 740 (2015).

[17] The $R$ factor describes the average match between measured $\left(F_{\text {obs }}\right)$ and calculated $\left(F_{\text {calc }}\right)$ diffraction peak intensity: $R=100$. $\sum_{i}\left(\left|F_{\text {obs }}\right|-\left|F_{\text {calc }}\right|\right) / \sum_{i}\left|F_{\text {obs }}\right|$.

[18] P. Wadley, A. Crespi, J. Gázquez, M. A. Roldán, P. García, V. Novak, R. Campion, T. Jungwirth, C. Rinaldi, X. Martí, V. Holy, C. Frontera, and J. Rius, J. Appl. Cryst. 46, 1749 (2013).

[19] A. N. Nateprov, V. Ch. Kravtsov, V. Fritsch, and H. von Löhneysen, Surf. Eng. Appl. Electrochem. 47, 540 (2011).

[20] D. E. Aspnes and A. A. Studna, Phys. Rev. B 27, 985 (1983).

[21] K. Uhlíŕová, R. Tarasenko, F. J. M. Casado, B. Vondráčková, and Z. Matěj, J. Alloys Compd. 650, 224 (2015).

[22] E. Emmanouilidou, H. Cao, P. Tang, X. Gui, C. Hu, B. Shen, J. Wu, S.-C. Zhang, W. Xie, and N. Ni, Phys. Rev. B 96, 224405 (2017).

[23] P. Blaha, K. Schwarz, G. K. H. Madsen, D. Kvasnicka, and J. Luitz, WIEN2k, An Augmented Plane Wave + Local Orbitals Program for Calculating Crystal Properties (Karlheinz Schwarz, Techn. Universität Wien, Austria), 2001.

[24] C. Ambrosch-Draxl and J. O. Sofo, Comput. Phys. Commun. 175, 1 (2006).

[25] T. Berlijn, D. Volja, and W. Ku, Phys. Rev. Lett. 106, 077005 (2011).

[26] V. I. Anisimov, I. V. Solovyev, M. A. Korotin, M. T. Czyzyk, and G. A. Sawatzky, Phys. Rev. B 48, 16929 (1993).

[27] R. Bertacco, M. Cantoni, M. Riva, A. Tagliaferri, and F. Ciccacci, Appl. Surf. Sci. 252, 1754 (2005).

[28] M. Cantoni and R. Bertacco, Rev. Sci. Instrum. 75, 2387 (2004).

[29] S. Hüfner, Photoelectron Spectroscopy (Springer, Heidelberg, 2003).

[30] J. Železný, Ph.D. thesis, MFF UK, Universitas Carolina, Praha, 2016, doi:10.13140/RG.2.1.4579.4800.

[31] J. Braun, K. Miyamoto, A. Kimura, T. Okuda, M. Donath, H. Ebert, and J. Minár, New J. Phys. 16, 015005 (2014).

[32] J. B. Pendry, Surf. Sci. 57, 679 (1976).

[33] J. Minár, H. Ebert, G. Ghiringhelli, O. Tjernberg, N. B. Brookes, and L. H. Tjeng, Phys. Rev. B 63, 144421 (2001).

[34] J. Braun, J. Minár, S. Mankovsky, V. N. Strocov, N. B. Brookes, L. Plucinski, C. M. Schneider, C. S. Fadley, and H. Ebert, Phys. Rev. B 88, 205409 (2013).

[35] H. Ebert, D. Ködderitzsch, and J. Minár, Rep. Prog. Phys. 74, 096501 (2011).

[36] G. Malmström and J. Rundgren, Comput. Phys. Commun. 19 , 263 (1980). 